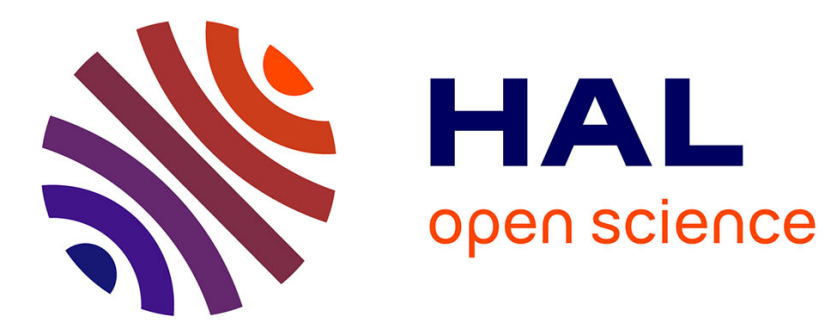

\title{
INTRODUCTION A L'UNIFICATION DES INTERACTIONS ELECTROMAGNETIQUES ET FAIBLES
}

\author{
F. Martin
}

\section{- To cite this version:}

F. Martin. INTRODUCTION A L'UNIFICATION DES INTERACTIONS ELECTROMAGNETIQUES ET FAIBLES. Journal de Physique Colloques, 1980, 41 (C3), pp.C3-99-C3-113. 10.1051/jphyscol:1980314 . jpa-00219833

\section{HAL Id: jpa-00219833 https://hal.science/jpa-00219833}

Submitted on 1 Jan 1980

HAL is a multi-disciplinary open access archive for the deposit and dissemination of scientific research documents, whether they are published or not. The documents may come from teaching and research institutions in France or abroad, or from public or private research centers.
L'archive ouverte pluridisciplinaire HAL, est destinée au dépôt et à la diffusion de documents scientifiques de niveau recherche, publiés ou non, émanant des établissements d'enseignement et de recherche français ou étrangers, des laboratoires publics ou privés. 


\title{
INTRODUCTION A L'UNIFICATION DES INTERACTIONS ELECTROMAGNETIQUES ET FAIBLES
}

\author{
F. MARTIN \\ CERN, 1211 Genève 23, Suisse.
}

\begin{abstract}
Résumé.-Après avoir décrit 1'état actuel de la phénoménologie des intéractions faibles on discute les principes de base des théories de jauge. On montre ensuite comment le mécanisme de Higgs permet d'obtenir des quanta d'intéraction massifs. Le modèle dit de "Weinberg-Salam" permettant d'unifier les intéractions électromagnétiques et faibles est présenté. On termine par quelques mots sur 1 'unification avec les intêractions fortes et la gravitation.
\end{abstract}

Abstract.-After reviewing the present status of weak interaction phenomenology we discuss the basic principles of gauge theories. Then we show how Higgs mechanism can give massive quanta of interaction. The so-called "Weinberg-salam" model, which unifies electromagnetic and weak interactions, is described. We conclude with a few words on unification with strong interactions and gravity.

1. Introduction.-L'unification des forces apparaissant dans la nature est une activité importante de la physique. Deux exemples célèbres en témoignent : tout d'abord Newton qui en 1687 a unifié la gravitation terrestre (chute des pommes) avec la gravitation céleste (mouvement des planètes autour du soleil). I'autre exemple est l'unification de l'électricité et au magnétisme réalisêe par Maxwell à la fin du lgème siècle. La situation actuelle est que l'on observe quatre types de forces, apparaissant comme différentes, qui régissent toutes les intéractions de la matière avec elle-même : la gravitation l'électromagnétisme, l'intéraction faible (qui se manifeste dans la radioactivité) et l'intéraction forte ou nucléaire (qui lie les protons et les neutrons à l'intérieur des noyaux). Notons que la gravitation telle que l'a formulée Newton a été transformée par Einstein en 1916 de manière à y inclure les principes de la relativité restreinte; cela constitue ce que l'on appelle la relativitê génêrale. De plus l'électromagnétisme telle que l'a formulé Maxwell a aussi été transformé de manière à y inclure les principes de la mécanique quantique. Cela a été fait entre 1926 et 1949 et constitue ce que l'on appelle l'électrodynamique quantique. Un des buts de la physique actuelle est de montrer que les quatre forces énoncées ci-dessus sont quatre aspects d'une seule et même force fondamentale. Un des schémas possibles d'unification est le suivant : $U(n)$ désigne le groupe des matrices unitaires $n$ sur $n ; s U(n)$ est le sous-groupe de ces matrices qui sont de déterminant 1 (spécial). Les points d'interrogations placés à côté de $S U(2) \times U(1)$ et de SU(3) couleur signifient que ces deux théories ont probablement quelque chose à voir avec la réalité mais que leurs preuves définitives restent encore à être trouvées. Quant aux doubles points d'interrogations qui suivent $\mathrm{SU}(5)$ et la supergravité, ils signifient que ces deux théories sont simplement des exemples de solutions et que dans l'état actuel de l'observation expérimentale il n'a pas encore été possible de les tester.

Le sujet de cet exposé est l'unification des intêractions électromagnétiques et faibles. Pour faire cet exposé je me suis grandement inspiré d'Abers et Lee $/ 1 /$, de Weinberg $/ 2 /$ et d'Iliopoulos $/ 3 /$. Le plan en est le suivant.' Après avoir fait état de la situation des intéractions faibles avant unification (paragraphe 2), j'introduirai le concept de thérie de jauge qui semble etre aujourd'hui à la basa de toutes les intéractions observées dans la nature et donc de leur unification (par. 3). 


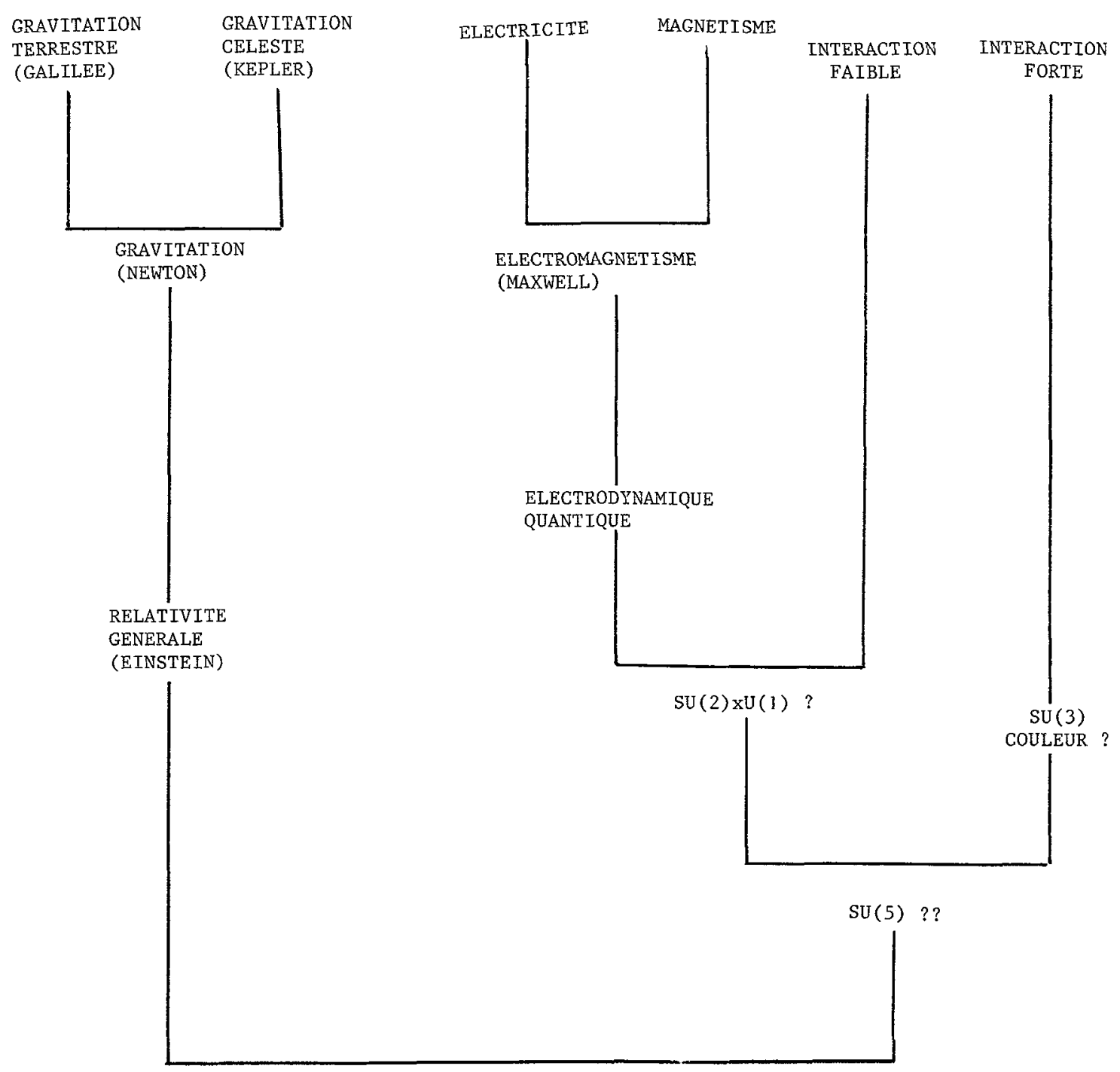

SUPERGRAVITE ??

Je montrerai ensuite comment on peut briser spontanément la symétrie de jauge, c'est-àdire obtenir des états physiques non symétriques tout en préservant la symétrie des équations fondamentales de la théorie (par.4) Le mođèle dit de "Weinberg-Salam", qui traite les intéractions électromagnétiques et faibles dans une théorie de jauge $\mathrm{SU}(2) \times \mathrm{U}(1)$ brisée spontanēment, fera 1 'objet du paragraphe 5. Le paragraphe 6 contient des conclusions et quelques mots sur l'unification avec les deux autres intéractions : l'intêraction forte et la gravitation.

2. Phénoménologie des intéractions faibles avant unification. - L'histoire des intéractions faibles commence en 1896 lorsque Becquerel découvrit la radioactivité de sels d'uranium. L'intéraction faible est responsable de la désintégration $\beta$ des noyaux radioactifs basêe, sur le processus

$$
\mathrm{n} \longrightarrow \mathrm{p}+\mathrm{e}^{-}+\bar{v}
$$

(est aussi pour certains noyaux sur le 
processus $\left.p \rightarrow n+e^{+}+v\right)$. C'est pour dêcrire ce processus qu'en 1934 Fermi proposa un modèle d'intéraction locale entre les quatre particules. Plutôt que de décrire le modèle de Fermi pour la désintégration $\beta$ du neutron je vais en présenter la version 1979. Pour cela il faut travailler avec les particules qui sont considérées comme élémentaires en 1979. Quelles sont ces particules élémentaires ? (Voir aussi l'exposé de F. Vanucci). Il $\mathrm{y}$ a tout d'abord les leptons dont la caractéristique est d'être insensibles aux forces nucléaires : électron, muon, lepton $\tau$ et leurs neutrinos correspondants $\left(e^{-}, \nu_{e}\right)$ $\left(\mu^{-}, \nu_{\mu}\right)\left(\tau^{-}, \nu_{\tau}\right)$. Le neutrino $\nu_{\tau} n^{\prime}$ 'a encore pas été directement mis en évidence, mais l'êtude de la désintégration du lepton $\tau$ semble bien impliquer son existence, avec les mêmes caractéristiques que $\nu_{e}$ et $\nu_{\mu}$, en particulier il serait aussi de masse nulle. A ces six leptons il faut aussi ajouter leurs six antiparticules $: e^{+}, \bar{\nu}_{e^{\prime}} \mu^{+}, \bar{\nu}_{\mu}$ $\tau^{+}$et $\bar{v}_{\tau}$. Jusqu'à présent ces leptons se sont comportés comme des particules ponctuelles c'est-à-dire qu'ils n'ont manifesté aucunè structure. Il y a ensuite les hadrons dont la caractéristique est d'être sensibles aux forces nucléaires. Tous les hadrons que l'on peut observer isolément dans les laboratoires, comme le proton et le neutron, ont une structure. On pense qu'ils sont constitués de particules plus élémentaires : les quarks. Mais si beaucoup de faits expérimentaux semblent impliquer leur existence, ils n'ont encore pas pu être isolés en laboratoire. La croyance actuelle est que 1 'on ne pourra jamais les isoler, qu'ils sont confinés à l'intérieur des hadrons observables (voir 1 'exposé de J. Iliopoulos).

A présent on pense avoir observé l'existence de cing "saveurs" différentes de quarks : $(d, u)(s, c)(b, t)$. L'existence du quark $t$ n'a pas encore été prouvée expéximentalement mais il $y$ a beaucoup de présomptions theoriques pour qu'il existe. De plus pour chaque saveur le quark possède aussi trois couleurs (voir l'exposé de $\mathrm{E}$. de Rafael) et il faut ajouter tous les antiquarks. Leptons et quarks sont des particules de spin $1 / 2$ (le spin est le moment angulaire intrinsèque $d$ 'une particule). Pour complèter ce tableau des particules élémentaires "observées" en 1979 il faut ajouter le photon qui lui est de spin 1.

Dans le modèle de Fermi l'intéraction faible est traitée comme une intéraction de type courant-courant. Ainsi il y a une intéraction courant chargé-courant chargê dont la contribution au Lagrangien s'écrit : $\mathscr{L}_{\mathrm{CC}}=\frac{\mathrm{G}}{\sqrt{2}} \mathrm{~J}_{\rho}(\mathrm{X}) \mathrm{Jp}^{\dagger}(\mathrm{X})$

Rappelons que le Lagrangien, ou pour être plus précis la densité de Lagrangien, est unefonction $\mathscr{\Psi}\left(\Phi_{i}(X), \partial_{\rho} \Phi_{i}(X)\right)$ des champs $\Phi_{i}(X)$ qui apparaissent dans la théorie ainsi que de leurs dérivées $\partial_{p} \Phi_{i}(x)$.

Le Lagrangien permet d'obtenir les équations du mouvement à 1 'aide des équations d'EulerLagrange :

$$
\frac{\partial \mathcal{L}}{\partial \Phi_{i}(X)}=\partial_{\rho} \frac{\partial \mathscr{L}}{\partial\left[\partial_{\rho} \Phi_{i}(X)\right]}
$$

$\mathrm{X}$ désigne un point d'espace-temps à ouatre composantes $x^{\rho}(\rho=0,1,2,3): x^{\circ}=c t$, $\left(\mathrm{x}^{1}, \mathrm{x}^{2}, \mathrm{x}^{3}\right)=\overrightarrow{\mathrm{x}}$. Les notations suivantes sont utilisées $:{ }_{\rho}=\frac{\partial}{\partial x_{\rho}}$ et $a_{\rho} b^{\rho}=a^{\circ} b^{\circ}-$
$-\vec{a} \vec{b}$.

Le courant chargé $J_{\beta}(x)$ de la formule (I) possède une partie leptonique $\ell_{\rho}(X)$ et une partie hadronique $h_{\rho}(x)$ :

$$
J_{\rho}(X)=l_{\rho}(X)+h_{\rho}(X)
$$

La partie leptonique s'écrit :

$$
\begin{aligned}
& \ell_{\rho}(x)=2 \bar{\psi}_{L}^{e}(x) \gamma_{\rho} \psi_{L}^{\nu_{e}}(x)+2 \bar{\psi}_{L}^{\mu}(x) \\
& \gamma_{\rho} \psi_{I}^{\nu}{ }^{\mu}(x)+2 \bar{\psi}_{L}^{\tau}(x) \gamma_{\rho} \psi_{L}^{\nu}{ }^{\tau}(x)
\end{aligned}
$$

Cette écriture justifie l'adjectif chargé donné au courant $J_{\rho}(x)$ car elle fait intervenir des couples de particules de charges différentes $\left(e \cdot g \cdot\left(e^{-}, \nu_{e}\right)\right) \cdot \psi(X)$ désigne un spineur de Dirac, c'est-à-dire une colonne à quatre composantes permettant de tenir compte en même temps du spin $1 / 2$ de la particule (deux degrés de libertē) et de son antiparticule (deux autres degrés de liberté). $\gamma_{\rho}$ désigne les quatre matrices de Dirac qui sont des matrices $\overline{4}$ sur 4 . $\bar{\psi}(\mathrm{X})=\psi^{\dagger}(\mathrm{X}) \gamma_{\mathrm{o}}$ est une ligne à quatre composantes. $\psi_{L}(\mathrm{X})$ signifie que seule la par- 
tie gauche ( $L=1$ eft) du spin de la particule intervient dans le courant faible chargé. Lorsqu'on néglige la masse de la particule la partie gauche correspond au spin pointant dans la direction opposée au mouvement de la particule. Le fait que seules les parties gauches des particules interviennent dans l'intéraction courant chargé-courant chargé implique une propriété importante des intéractions faibles : elles violent la parité. En effet la parité est la transformation qui change $\vec{X}$ en $-\vec{X}$ et dans cette transformation les parties gauches se changent en parties droites. On voit donc que le Lagrangien (1) n'est pas invariant par parité. Une autre propriété du Lagrangien (1) est qu'il représente une intéraction locale à quatre particules. En effet si nous considérons le terme qui correspond à la désintégration du $\mu^{-}\left(\mu^{-} \rightarrow e^{-}+\nu_{\mu}+\bar{v}_{e}\right):$

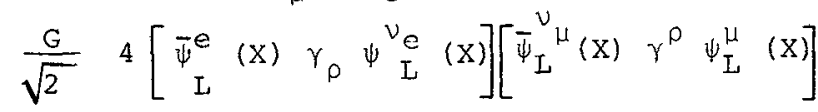
nous voyons que ce terme implique que les quatre particules interagissent au même point d'espace-temps $x$.

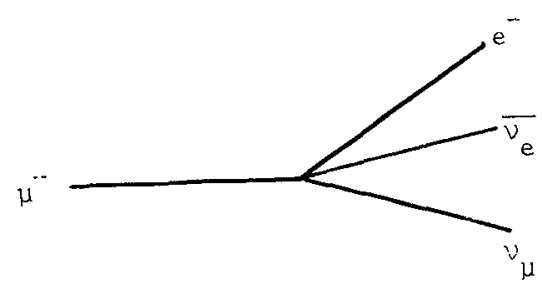

Ia constante $G$ qui apparait dans la formule (1) est appelée constante de couplage de Fermi :

$$
G=1,01 \frac{10^{-5}}{m_{p^{2}}^{2}}
$$

où $\mathrm{m}_{\mathrm{p}}$ est la masse du proton. La petitesse de cette constante de couplage justifie l'adjectif faible qui dêsigne cette intéraction. Ia partie hadronique du courant $J_{\rho}(X)$ s'écrit

$$
\begin{aligned}
h_{\rho}(\mathrm{X})= & 2 \bar{\psi}_{\mathrm{L}}^{\mathrm{u}}(\mathrm{X}) \gamma_{\rho} \psi_{\mathrm{L}_{\theta_{1}}}(\mathrm{x}) \\
& +2 \bar{\psi}_{\mathrm{L}}^{\mathrm{c}}(\mathrm{X}) \gamma_{\rho} \psi_{\mathrm{S}_{\theta_{1}}}(\mathrm{x}) \\
& +\ldots
\end{aligned}
$$

où

$$
\left\{\begin{array}{l}
\psi^{\mathrm{d}_{\theta_{1}}}(\mathrm{x})=\cos \theta_{1} \psi^{\mathrm{d}}(\mathrm{x})+\sin \theta_{1} \psi^{\mathrm{s}}(\mathrm{x}) \\
\psi_{\mathrm{s}_{2}}(\mathrm{x})=-\sin \theta_{1} \psi^{\mathrm{d}}(\mathrm{x})+\cos \theta_{1} \psi^{\mathrm{s}}(\mathrm{x})
\end{array}\right.
$$

$\theta_{1}$ est appelé angle de Cabibbo, il est relativement petit $\left(\sin \theta_{1} \sim 0,24\right)$. Le courant hadronique $h_{p}(x)$ tel qu'il est écrit cidessus, faisant intervenir le quark charmé c, a êté formulé en 1970 par Glashow, Iliopoulos et Maiani. Si en plus nous faisons intervenir les quarks b et $t$ nous obtenons des mélanges un peu plus compliqués.

Le modèle de Fermi tel qu'il vient d'être présentê (avec l'adjonction d'un terme d'intéraction courant neutre-courant neutre où neutre signifie que le courant fait intervenir des couples de particules de même charge électrique), permet d'expliquer toute la phénoménologie des intéractions faibles observée actuellement. Ceci en utilisant l'intéraction de Fermi au premier ordre. Mais le modèle de Fermi n'est pas une thêorie. En effet premièrement on ne peut faire des calculs qu'au premier ordre de 1'intéraction. Les corrections d'ordres supérieurs n'ont pas de sens. Deuxièmement le premier orare n'est pas valable à toutes les énergies. Au-dessus d'une certaine énergie il y a violation du principe a'unitarité, c'est-à-dire de la conservation de la probabilité.

Comparons le modèle de Fermi avec une théorie déjà connue : l'électrodynamique quantique. La contribution de l'intéraction électromagnétique de l'électron au Lagrangien est

$$
\mathcal{L} \text { em }=e\left[\begin{array}{lll}
\bar{\psi} & e(x) & \gamma_{\rho} \psi^{e}(x)
\end{array} A^{\rho}(x)\right.
$$

où $A^{\rho}(X)$ est le champ électromagnétique et -e <0 la charge de l'électron. Nous voyons qu'ici il s'agit d'une intêraction locale courant-champ électromagnétique :

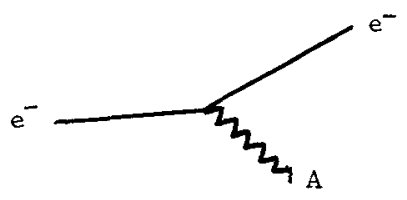

Ecrivons l'intéraction électromaọnétique entre deux électrons à l'ordre le plus bas. Pour cela il faut considérer le diagramme de Feynman suivant : 


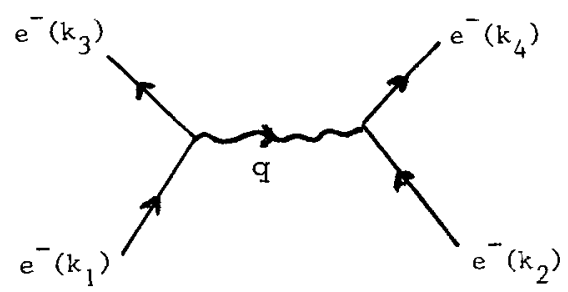

qui dans 1 'espace des moments donne un terme d'intéraction de la forme

$-\frac{e^{2}}{q^{2}}\left[\bar{\psi}^{e}\left(k_{3}\right) \gamma_{\rho} \psi^{e}\left(k_{1}\right)\right]\left[\bar{\psi}^{e}\left(k_{4}\right) \gamma^{\rho} \psi^{e}\left(k_{2}\right)\right]$ oì $\mathrm{q}=\mathrm{k}_{3}-\mathrm{k}_{1}$ est le moment de transfert $\left(\mathrm{q}^{2}<0\right)$.

Il s'agit d'un terme d'intéraction du type courant-courant comme dans la formule (1) mais oũ le terme multiplicatif n'est pas une constante comme $\frac{4 G}{\sqrt{2}}$ mais une fonction de $\mathrm{q}^{2}$ : $\frac{e^{2}}{-q^{2}}$. Par analogie imaginons donc que 1 'intéraction faible de type courant chargé se fasse aussi via l'échange d'un quanta de spin 1 (et de masse $m_{W}$ ) :

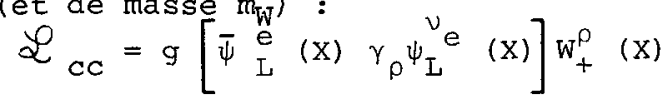

+ conjugue hermitique $+\ldots$.

Comme dans le cas de l'électromagnétisme nous avons une intéraction locale courantchamp vectoriel représentée par le diagramme élémentaire :

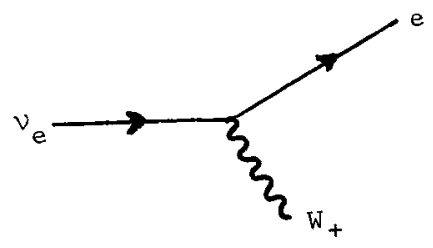

Ecrivons de même l'intëraction $v_{e} e^{-} \rightarrow \nu_{e} e^{-}$ à l'ordre le plus bas :

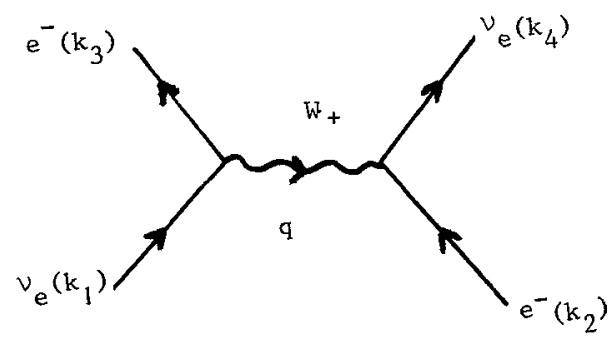

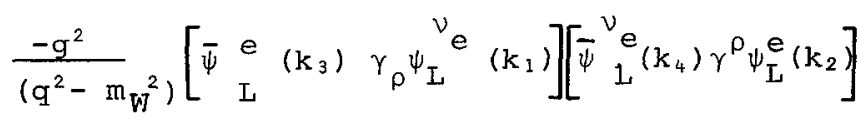

Comme dans le cas de l'électromagnétisme il s'agit d'une intêraction courant-courant où le terme multiplicatif est fonction de $q^{2}$.
Mais lorsque $\mathrm{q}^{2}$ est petit devant $\mathrm{m}_{\mathrm{W}}{ }^{2}$ nous pouvons négliger la dépendance en $\mathrm{q}^{2}$ et nous retrouvons le modèle de Fermi avec

$$
\frac{4 \mathrm{G}}{\sqrt{2}}=\frac{\mathrm{g}^{2}}{\mathrm{~m}_{\mathrm{w}}^{2}}
$$

Fait important, le facteur $\frac{-1}{\mathrm{q}^{2}-m_{\mathrm{q}_{2}}^{2}}$ qui est différent de $\frac{1}{m_{T}^{2}}$ pour $q^{2} \gg m_{i N}$ résout le problème, énóncé pius haut, de la violation de l'unitarité du modèle de Fermi. Si dans le cadre d'une unification des intéractions électromagnétiques et faibles nous

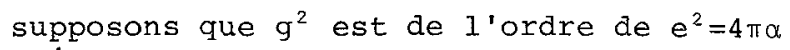
$=\frac{4 \pi}{137} \quad$ la formule (7) nous dit que $m_{W}$ est alors de l'ordre de $50 \mathrm{GeV}$. Ce chiffre est tout à fait raisonnable car expérimentalement on n'a encore pas mesuré de processus oũ les transferts sont de cet ordre.

Nous sommes sur le chemin d'une théorie des intéractions faibles mais nous n'en avons pas pour autant terminé. En effet si l'introduction du boson $W$ permet de résoudre un des problèmes du modèle de Fermi : la violation de l'unitarité, par contre le calcul des termes d'ordres supérieurs à $G$ (ou $\mathrm{g}^{2}$ ) n'a toujours pas de sens. Reprenons 1 'exemple de l'électrodynamique quantique. On ne sait pas résoudre le problème de 1 'intêraction électromagnétique de manière complète et globale mais seulement de manière perturbative, c'est-à-dire ordre par ordre en la constante de couplage e. Par exemple, le terme représenté par le diagramme de Feynman suivant :

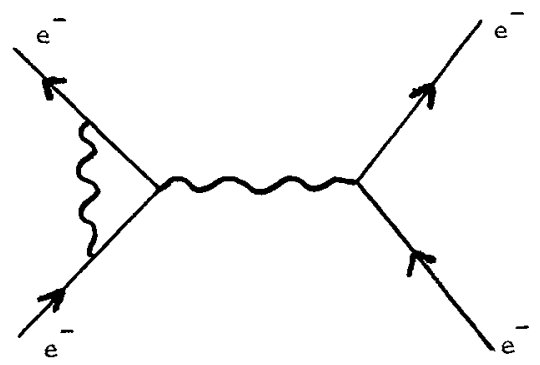

est un terme d'ordre $e^{4}$ pour le processus $e^{-} e^{-} \longrightarrow e^{-} e^{-}$. L'électrodynamique quantique possède deux propriétés qui en font une théorie que l'on peut tester expérimentalement. (a) Elle est renormalisable ; ceci signifie que le calcul d'un ordre quelconque en la constante de couplage e a un sens. (b) La constante de couplage e est 
faible; il en rêsulte que les termes de la série de perturbation sont de plus en olus petits à mesure que l'ordre augmente. De plus c'est une théorie qui est en très bon accord avec l'expérience. Elle a êté testée avec une très grande précision (de l'ordre de $10^{-7}$ pour le moment magnétique de I'électron). L'intéraction faible formulée avec échanges de boson $W$ possède la propriété (b) mais ne possède pas la propriété (a). En effet si l'électrodynamique quantique est renormalisable c'est parce que le photon, quanta du champ électromagnétique, est de masse nulle. Par contre le boson $W$, quanta des intéractions faibles, ne peut être que très massif. Ceci empêche la renormalisabilité d'une thêorie basée seulement sur le Lagrangien (6). La solution de ce problème est de considérer les théories de jauge avec brisure spontanée de symêtrie.

Ceci va être l'objet des deux prochains paragraphes. Avant de passer à ceux-ci résumons brièvement la comparaison entre les intéractions faibles et électromagnétiques. Il y a tout d'abord une similitude : toutes les deux peuvent apparaitre comme des intéractions courant-courant. Ceci ne serait que le reflet d'intéractions locales courantquanta de spin 1. Il y a ensuite des différences : l'intéraction faible est beaucoup plus faible que l'intéraction électromagnétique aux énergies actuelles. De plus, à ces mêmes énergies l'intéraction faible apparât comme de portée nulle alors que l'intéraction électromagnétique est de portée infinie. Ces deux différences trouvent leur explication dans les masses des quanta d'intéraction :

$$
m_{W} \approx 50-100 \mathrm{GeV}, m_{\gamma}=0 \text {. }
$$

Il $y$ a encore d'autres différences : l'intéraction faible fait intervenir des courants chargés et neutres alors que l'intéraction électromagnétique ne fait intervenir que des courants neutres. De plus l'intéraction faible viole la parité aloxs que l'intéraction électromagnétique ést invariante par parité. Si toutes ces différences apparaissent comme importantes, en fait elles n'empêchent pas de formuler une théorie des intéractions faibles et même d'unifier les deux intêractions.
3. Les théories de jauge.-a) Nous allons tout d'abord considérer le cas le plus simple d'une particule de spin 0. Pour cela étudions la densité de Lagrangien d'un champ scalaire complexe libre, décrivant en même temps la particule et son antipar$\mathscr{L}^{\text {ticule }}=\left[\partial^{\rho} \Phi(\mathrm{X})\right]^{*} \partial_{\rho} \Phi(\mathrm{X})-\mathrm{m}^{2} \Phi^{*}(\mathrm{X}) \Phi(\mathrm{X})$ Les équations d'Euler - Lagrange (2) permettent d'obtenir l'équation du mouvement de ce champ libre :

$$
\partial_{\rho} \partial^{\rho} \Phi(x)+m^{2} \Phi(x)=0
$$
appelée équation de Klein-Gordon, où $m$ est la masse de la particule. Le Lagrangien (8) est invariant par la transformation

$$
\Phi(\mathrm{X}) \longrightarrow \mathrm{e}^{\mathrm{i} \Theta_{\Phi}(\mathrm{X})}
$$

où $\theta$ est un nombre réel. L'ensemble des nombres complexes de la forme $e^{i \theta}$ constitue le groupe $U(1)$. On dit que le Lagrangien et par conséquent la théorie sont invariants par les transformations globales du groupe $U(1)$, globales $\operatorname{car} \theta$ est le même en tout point de l'espace-temps. Considérons maintenant des transformations locales, où $\theta$ est différent en chaque point de 1 'espacetemps :

$$
\Phi(\mathrm{X}) \longrightarrow e^{i \Theta(X)} \Phi(\mathrm{X})
$$

Le Lagrangien (8) n'est pas invariant par ces transformations car

$$
\partial_{\rho} \Phi(x) \rightarrow e^{i \theta(x)} \partial_{\rho} \Phi(x)+i \partial_{\rho} \theta(x) e^{i \theta(x)} \Phi(x)
$$

$S i$ nous voulons avoir un Lagrangien invariant par ces transformations locales il est nécessaire d'introduire une intéraction du champ scalaire $\Phi(X)$ avec un champ vectoriel $A_{\rho}(X)$. Pour cela on remplace dans le Lagrangien (8) la dérivée usuelie a par

$$
D_{p}=\partial_{\rho} \text { - ie } A_{p}(X)
$$

que l'on appelle dérivée covariante. De plus on postule que les transformations locales du groupe $U(1)$ modifient le champ $A_{\rho}(X)$ de la manière suivante :

$$
A_{\rho}(X) \longrightarrow A_{\rho}(X)+\frac{1}{e} \partial_{\rho} \Theta(X)
$$

Remarquons que cette transformation est la transformation de jauge familière en électromagnétisme : on ajoute au potentiel vecteur, dont dérive les champs électrique et magnêtique, le gradient d'un champ scalaire arbitraire, ce qui ne modifie pas 
les équations de Maxwell. Suivant les transformations locales (11) et (13)

$D_{\rho} \Phi(X)=\partial_{\rho} \Phi(X)$ - ie $A_{\rho}(X) \Phi(X)$ se transforme en

$$
\begin{aligned}
& e^{i \Theta(X)} \partial_{\rho} \Phi(X)+i \partial_{\rho} \Theta(X) e^{i \Theta(X)} \Phi(X) \\
& -i e A_{\rho}(X) e^{i \Theta(X)} \Phi(X)-i \partial_{\rho} \Theta(X) e^{i \Theta(X)} \Phi(X) \\
& =e^{i \Theta(X)} D_{\rho} \Phi(X)
\end{aligned}
$$

Par conséquent le Lagrangien

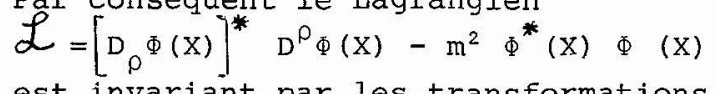

est invariant par les transformations locales (11) et (13) du groupe $U(1)$. Il faut de plus ajouter à ce Lagrangien le terme cinétique du champ vectoriel :

$$
-\frac{1}{4} F^{\rho \sigma}(X) \quad F_{\rho \sigma}(X)
$$
où $F^{\rho \sigma}(X)=\partial^{\rho} A^{\sigma}{ }^{\rho \sigma}(x)-\partial^{\sigma} A^{\rho}(x)$. Il est facile de voir que ce terme est invariant par les transformations locales (13). Remarquons que c'est ce terme qui permet d'obtenir les équations de Maxwell et que $F^{\rho \sigma}(X) n^{\prime}$ 'est autre que la représentation covariante des champs électrique et magnétique :

$\overrightarrow{\mathrm{E}}=\left(\mathrm{F}^{10}, \mathrm{~F}^{20}, \mathrm{~F}^{30}\right)$

$\vec{B}=-\left(F^{23}, F^{31}, F^{12}\right)$
Il est important de remarquer que si le terme (15) est invariant par les transformations locales du groupe $U(1)$, par contre un terme de masse non nulle du champ vectoriel :

$$
\frac{1}{2} M^{2} A_{\rho}(X) A^{\rho}(X)
$$

$n$ 'est pas invariant par ces transformations locales. Le Lagrangien (14) auquel on ajoute le terme (15) constitue le Lagrangien de I'intéraction électromagnétique d'une particule de spin 0 et de charge -e $<0$ (par exem. ple le $\pi^{-}$).

Les transformations locales associées à une symétrie globale sont aussi appelées transformations de jauge. Quant aux théories invariantes par ces transformations locales on les appelle théories de jauge. Une image va nous permettre d'illustrer ce qu'est une théorie de jauge. Considérons une sphère (ou un ballon) sur laquelle un système de méridiens et de parallèles permet de repérer les points de la surface /4/.

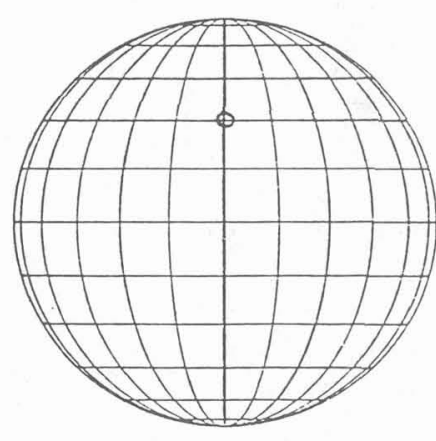

sphère originale

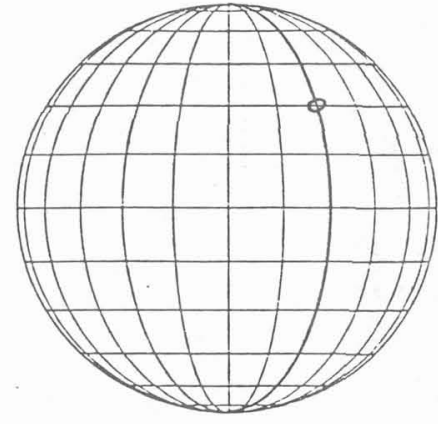

transformation globale

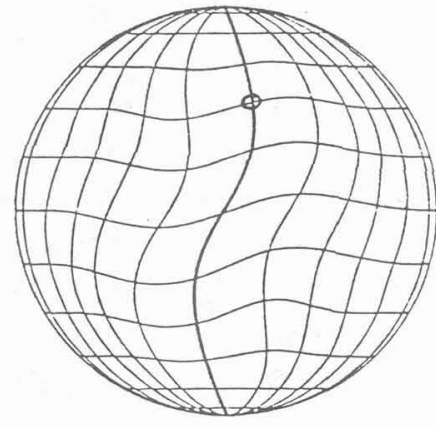

transformation locale

Une rotation d'un angle $\theta$ autour d'un des modifiêe, en particulier les transformaaxes de la sphère est une transformation globale; en effet chaque point de la sphère subit la même rotation. La sphère est invariante par cette rotation. De plus la surface de la sphère n'est pas modifiée; on a simplement "globalement" tourné la sphère. Maintenant considérons une transformation locale pour laquelle l'angle et l'axe de rotation sont différents en chaque point de la sphère. La forme de la sphère en tant que sphère ne change pas, mais par contre la surface est tions locales introduisent des tensions, des forces entre les points à la surface de la sphère (s'imaginer un ballon en caoutchouc). Cette image est la base des théories de jauge. On peut penser que toutes les forces qui régissent le comportement de la matière sont de ce type. On sait déjà qu'au moins deux des quatre forces actuellement observées dans la nature le sont : - l'électromagnétisme pour lequel les transformations de jauge forment le groupe 
$U(1)$.

- la gravitation pour laquelle les transformations de jauge constituent le groupe de Poincaré.

Si une invariance globale par le groupe de Poincaré engendre la relativité restreinte, une invariance locale engendre elle la relativité générale.

Il semble de plus que les intéractions faibles et les intëractions fortes soient aussi de ce type.

b) Complétons ce tableau des transformations de jauge en introduisant les particules de spin $1 / 2$ pour lesquelles le Lagrangien 1ibre est

$$
\mathcal{L}=\bar{\psi}(\mathrm{x})\left(i \gamma_{p} \partial^{p}-\mathrm{m}\right) \psi(\mathrm{X})
$$

Les équations d'Euler-Lagrange (2) conduisent à l'équation du mouvement suivante :

$$
\left(i \gamma_{\rho} \partial^{\rho}-m\right) \psi(X)=0
$$

qui est l'équation de Dirac, m étant la masse de la particule. De même que le Lagrangien (8), Ie Lagrangien (17) est invariant par les transformations globales du groupe $\mathrm{U}(1)$ :

$$
\psi(x) \longrightarrow e^{i \theta} \psi(x)
$$

Pour qu'il soit en plus invariant par les transformations locales de ce même groupe :

$$
\psi(\mathrm{x}) \longrightarrow \mathrm{e}^{\mathrm{i} \Theta(\mathrm{X})} \psi(\mathrm{x})
$$

il faut introduire une intéraction entre la particule de $\operatorname{spin} 1 / 2$ et un champ vectoriel $A_{\rho}(X)$. Pour cela on remplace la dérivée usuelle $\partial_{\rho}$ par la dérivée covariante (12) et on postule que le champ $A_{\rho}(X)$ se transforme suivant (13). Le Lagrangien localement invariant par le groupe $U(1)$ est alors $\mathscr{L}=\bar{\psi}$ (X) $\left(i \gamma_{\rho} D^{\rho}-m\right) \psi$ $(\mathrm{X})-\frac{1}{4} \mathrm{~F}^{\rho \sigma}$

(X) $F_{p \sigma}(X)(2$ Les équations d'Euler-Lagrange (2) permettent a'obtenir les équations du mouvement suivantes :

$$
\left\{\begin{array}{l}
\left(i \gamma_{\rho} \partial^{\rho}-m\right) \psi(x)=-e A^{\rho}(x) \gamma_{\rho} \psi(x) \\
\partial^{\sigma} F_{\rho \sigma}(x)=e \bar{\psi}(x) \gamma_{\rho} \psi(x)
\end{array}\right.
$$

qui sont les équations de l'intéraction électromagnétique de l'électron (charge $-e<0$ ).

c) Tout ceci peut être généralisé pour des groupes non abéliens, c'est-à-dire non commutatifs, comme par exemple su(2). Ceci a été fait en 1954 par Yang et Mills, $\Phi(X)$ ou $\psi(x)$ sont alors dans des espaces dans lesquels agit le groupe, ce sont des vecteurs colonnes. Les transformations globales s'écrivent :

$$
\Phi(\mathrm{X}) \longrightarrow \mathrm{e}^{\mathrm{i} \dot{\mathrm{\theta}} \overrightarrow{\mathrm{I}}} \Phi(\mathrm{X})=\mathrm{U}(\theta) \Phi(\mathrm{X}),
$$

de même pour $\psi(X)$. $\vec{L}$ représente un ensemble de matrices indëpendantes ( 3 pour $\mathrm{SU}(2)$ ) qui ne commutent pas nécessairement :

$$
\left[L_{j}, L_{k}\right]=i C_{j k l} L_{I}
$$

oũ les $C_{j k l}$ sont les constantes de structure du groupe $\left(C_{j k l}=\varepsilon_{j k l}\right.$ pour $\left.\mathrm{su}(2)\right)$. L'ensemble des $\vec{\theta} \vec{L}$ constitue ce oue $I$ 'on appelle l'algèbre de Lie du groupe.

Les transformations locales sont :

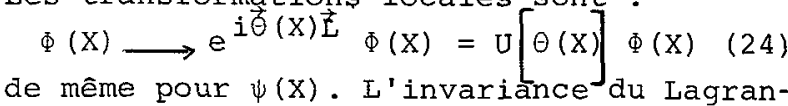
gien par ces transformations locales nécessite l'introduction d'un ensemble de champs vectoriels $\vec{A}_{\rho}(X)$ ( 3 pour $S U(2)$ ) et le remplacement des dérivées usuelles $\partial_{\rho}$ par des dérivées covariantes :

$$
D_{\rho}=\partial_{\rho}-\operatorname{ig} \vec{A}_{\rho}(X) \vec{I}
$$

où g est la constante de couplage de l'intéraction.

Les transformations locales modifient les champs $\overrightarrow{\mathrm{A}}_{\rho}(\mathrm{X})$ de la manière suivante :

$$
\begin{aligned}
\overrightarrow{\mathrm{L}} \vec{A}_{\rho}(X) \stackrel{\rho}{\longrightarrow} & U[\theta(X)] \overrightarrow{\mathrm{L}} \vec{A}_{\rho}(X) U^{-1}[\theta(X)] \\
& -\frac{i}{g}\left(\partial_{\rho} U[\theta(X)]\right) U^{-1}[\theta(X)]
\end{aligned}
$$

Les Lagrangienşinvariants de jauge s'écri-

$$
\begin{aligned}
\mathcal{L} & =\left[D_{\rho} \Phi(\mathrm{X})\right]^{\dagger}\left[\mathrm{D}^{\rho} \Phi(\mathrm{X})\right]-\mathrm{m}^{2} \Phi^{\dagger}(\mathrm{X}) \Phi(\mathrm{X}) \\
& -\frac{1}{4} \overrightarrow{\mathrm{F}}^{\rho_{\sigma}}(\mathrm{X}) \overrightarrow{\mathrm{F}}_{\rho_{\sigma}}(\mathrm{X})
\end{aligned}
$$

pour les particules de spin 0 , et $\mathscr{L}=\bar{\psi}(\mathrm{x}) \quad\left(\mathrm{i} \gamma_{\rho} D^{p}-\mathrm{m}\right) \quad \psi(\mathrm{x})-\frac{\mathrm{I}}{4} \overrightarrow{\mathrm{F}}^{\rho \sigma}(\mathrm{x}) \overrightarrow{\mathrm{F}}_{\rho \sigma}(\mathrm{x})$

pour les particules de spin $1 / 2$

$F_{j}^{P \sigma}(X)=\partial^{\rho} A_{j}^{\sigma}(X)-\partial^{\sigma} A_{j}^{\rho}(X)+$

$$
+g C_{j k l}+A_{k}^{\rho}(x) A_{l}^{\sigma}(X)
$$

Notons que comme précédemment des termes de masses non nulles des champs vectoriels, de la forme

$$
\frac{1}{2} M^{2} \vec{A}_{\rho}(X) \vec{A}^{\rho}(X)
$$

ne sont pas invariants de jauge.

Si nous examinons les Lagrangiens(27) et (28) nous nousapercevons que par rapport aux théories de jauge abéliennes apparaissent des termes supplére.ttaires d'intêractions trilinéaires et quadrilinéaires des 
champs vectoriels avec eux-mêmes :
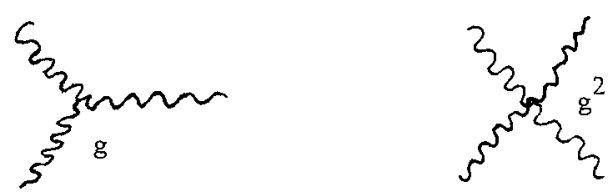

d) Un résultat est qu'une théorie, dans laquelle le Lagrangien est invariant par des transformations locales (ou transformations de jauge), est renormalisable. Mais une telle théorie nécessite, comme nous l'avons vu plus haut, que ses quanta d'intéraction soient de masses nulles. Or dans le cas des intéractions faibles les quanta d'intéraction sont massifs, il semble donc difficile d'inclure les intéractions faibles dans une théorie de jauge. Nous allons voir, dans le prochain paragraphe, qu'il est possible d'introduire des termes de masses non nulles pour les quanta d'intéraction tout en préservant $I^{\top}$ invariance de jauge du Lagrangien. Ceci est réalisé par la brisure spontanée de la symétrie de jauge. Un résultat, démontré par 't Hooft et Veltman en 1971, est que lorsqu'il y a brisure spontanēe de la symétrie de jauge, la théorie est encore renormalisable.

4. Brisure spontanée de la symétrie de jauge.Nous allons considérer le cas le plus simple d'une particule de spin 0 , avec l'invariance par le groupe $U(1)$.

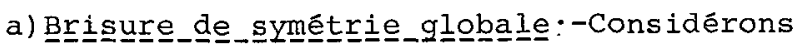
le Lagrangien (8) globalement invariant par le groupe $U(1)$. Récrivons le de la manière suivante :

$\mathcal{L}^{\prime}=\left[\partial^{\rho} \Phi(\mathrm{X})\right]^{*} \quad \partial_{\rho} \Phi(\mathrm{X})-\mathrm{V}(\Phi)$

avec $\mathrm{V}(\Phi)=\mathrm{m}^{2} \Phi^{*}(\mathrm{X}) \Phi(\mathrm{X})$

La densité d'Hamiltonien correspondante s'écrit $^{\prime}=\partial_{0} \Phi^{*}(X) \partial_{0} \Phi(X)+\sum_{j=1}^{3} \partial_{j} \Phi^{*}(X) \partial_{j} \Phi(X)+V(\Phi)$
(33) c'est elle qui permet d'obtenir l'énergie du système. L'état fondamental, c'est-à-dire le vide, correspond au niveau d'énergie minimum. Les deux premiers termes de la formule (33) sont positifs ou nuls, l'état fondamental correspond donc à $\Phi(X)$ indépendant de $X$ et $V(\Phi)$ minimum. Lorsque $V(\Phi)$ est donné par la formule (32), avec $\mathrm{m}^{2}>0$, le minimum de $V(\Phi)$ est obtenu pour $\Phi=0$ :

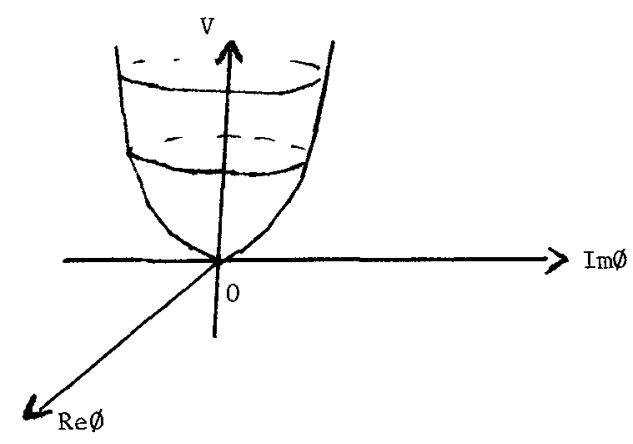

Le vide correspond donc à $\Phi(X)=0$. La particule de masse $m$ associée au champ $\Phi(X)$ a une probabilité de présence nulle dans le vide, ce qui est tout à fait raisonnable. De plus le vide $\Phi(X)=0$ est unique et est invariant par le groupe $U(1)$. Maintenant considérons $\mathrm{m}^{2}$ comme un paramètre réel et voyons si cela a un sens de le rendre négatif. Dans ce cas $V(\Phi)$ n'a pas de minimum fini, il est alors impossible de dêfinir un vide. Nous sommes dans une situation qui apparaît comme complètement non physique. Pour la rendre physiquement intéressante, modifions $V(\Phi)$ de la manière suivante :

$\mathrm{V}(\Phi)=\mathrm{m}^{2} \Phi^{*}(\mathrm{X}) \Phi(\mathrm{X})+\lambda\left(\Phi^{*}(\mathrm{X}) \Phi(\mathrm{X})\right)^{2}$

Nous avons rajouté au Lagrangien libre (31) un terme d'intéraction qui est, lui aussi, globalement invariant par le groupe $U(1)$ et qui ne modifie pas le caractère de renormalisabilité de la théorie.

Lorsque $\hat{\lambda}$ est positif nous nous retrouvons dans une situation où $V(\Phi)$ a un minimum fini :

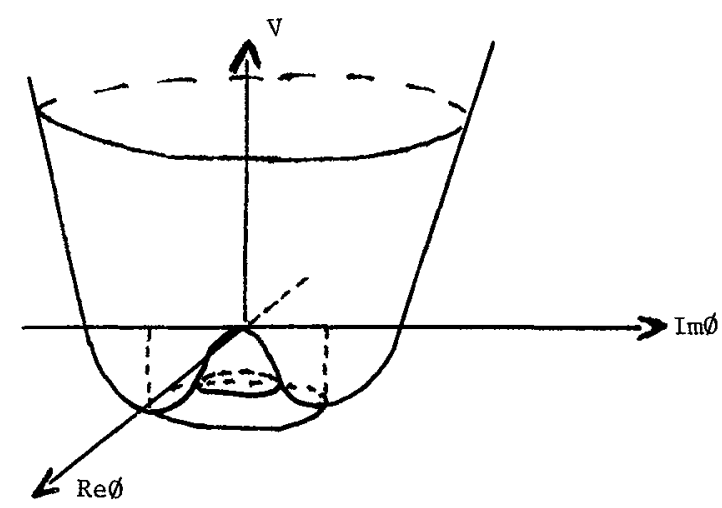

Mais dans ce cas le minimum d'énergie ne correspond plus à $\Phi(X)=0$ mais à toute la famille des $\Phi(X)$ de la forme : 


$$
\Phi(x)=e^{i \theta} \sqrt{\frac{-m^{2}}{2 \lambda}}
$$

Ainsi l'état fondamental, ou vide, est dégénéré.

De plus il n'y a pas de particule réelle correspondant au champ $\Phi(X)$. Ceci pour deux raisons : tout d'abord s'il $y$ en avait une elle aurait une probabilité de présence non nulle dans le vide, car d'après (35) le vide est associé à une valeur $\Phi(X) \neq 0$.

De plus elle aurait une masse carrée $\mathrm{m}^{2}$ nëgative, ce qui est non physique. En fait la manière de faire apparaitre la, ou les, particules associées au Lagrangien (31) (avec (34) et $\mathrm{m}^{2}<0$ ) est la suivante : on choisit

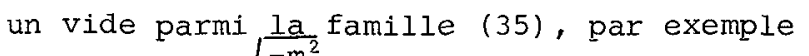

$$
\Phi_{0}=\sqrt{\frac{-m^{2}}{2 \lambda}}
$$

et $I^{\prime}$ 'on définit un nouveau champ

$$
\tilde{\Phi}(\mathrm{X})=\Phi(\mathrm{X})-\Phi_{0}
$$

tel que le vide corresponde à $\tilde{\Phi}(X)=0$

Il est alors nécessaire de séparer parties

réelle et imaginaire de $\tilde{\Phi}(\mathrm{X})$ :

$$
\tilde{\Phi}(\mathrm{X})=\frac{1}{\sqrt{2}}(\xi(\mathrm{X})+\operatorname{in}(\mathrm{X}))
$$

Le Lagrangien $(31)$, (34) s'écrit alors

$$
\begin{aligned}
\mathscr{L} & =\frac{1}{2} \partial_{\rho} \eta(x) \partial^{\rho} \eta(x)+\frac{1}{2} \partial_{\rho} \xi(x) \partial^{\rho} \xi(x)+m^{2} \xi^{2}(x) \\
& -\lambda \Phi_{0} \sqrt{2} \xi(x)\left(\xi^{2}(x)+n^{2}(x)\right) \\
& -\frac{\lambda}{4}\left(\xi^{2}(x)+n^{2}(x)\right)^{2}
\end{aligned}
$$

Si $\lambda$ est petit on peut traiter les deux derniers termes de manière perturbative, les trois premiers termes constituant la partie non perturbée. De ces trois premiers termes on peut alors extraire la structure en particule de la thêorie. Au champ $\xi(x)$ correspond une particule scalaire de masse $\sqrt{-2 \mathrm{~m}^{2}}$ et au champ $n(X)$ correspond une particule scalaire de masse nulle, dénommée boson de Goldstone. Remarquons que si par le changement de variable (37) et (38) nous avons fait apparaitre la structure en particule de la théorie, par contre nous avons perdu la symétrie manifeste du Lagrangien par le groupe U(1).

La brisure spontanée de symétrie correspond donc à un Lagrangien, c'est-à-dire à des équations du mouvement, qui sont invariants par un certain groupe de symétrie, alors que les états physiques, à commencer par le vide, ne sont pas invariants par ce groupe. Une image, très proche en fait de l'exemple qui a été consiđéré dans ce paragraphe, nous permet d'illustrer ceci. Il s'agit de la roulette. Les équations du mouvement de la roulette et de la bille sont invariantes dans toute rotation autour de l'axe de la roulette.

Malgré cela, à la fin du jeu la bille se retrouvera toujours dans une position asymêtrique.

Les caractéristiques fondamentales de la brisure spontanêe de symétrie sont les suivantes :

- la solution symétrique (ici $\Phi=0$ ) est instable

- I'état fondamental est dégênéré

- il existe un seuil critique à partir duquel apoarait la brisure de symétrie (ici $\mathrm{m}^{2}=0$ ). Dans de nombreux cas ce seuil correspond une température critique. Il y a beaucoup d'exemples de brisure spontanée de symétrie en physique : la cristallisation, le ferromagnétisme, la supraconđuctivitê,...

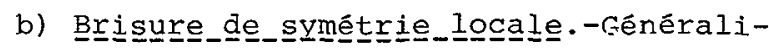
sons ce que nous venons de faire pour un Lagrangien localement invariant par le groupe U(1) :

$\mathcal{L}=\left(\partial^{\rho}+\right.$ ie $\left.A^{\rho}(X)\right) \Phi^{*}(X)\left(\partial_{\rho}-\right.$ ie $\left.A_{\rho}(X)\right) \Phi(x)$

$$
-V(\Phi)-\frac{1}{4} F^{\rho \sigma}(X) F_{\rho_{\sigma}}(X)
$$

Considérons tout de suite le cas ol $V(\Phi)$ est donné par (34) avec $\mathrm{m}^{2}<0$. Exactement comme dans le paragraphe précédent, la procédure pour faire apparaitre les particules associées à ce Lagrangien est tout d'abord de choisir un vide, par exemple

$$
\Phi_{0}=\sqrt{\frac{-m^{2}}{2 \lambda}}
$$

Ensuite il faut définir de nouveaux champs tels que le vide corresponde à l'annulation de ces nouveaux champs. Plutôt que de choisir le changement de variable (37-38), nous allons choisir le changement suivant :

$$
\Phi(X)=e^{i n(X)}\left(\Phi_{0}+\frac{1}{\sqrt{2}} \xi(X)\right)
$$

le vide correspond bien $\grave{a} n(x)=\xi(x)=0$. Puisque le Lagrangien (40) est invariart de jauge faisons la transformation de jauge correspondant à $\theta(x)=-n(x)$ Le nouveau champ vectoriel est alors donné par (13) :

$$
\tilde{A}_{\rho}(X)=A_{\rho}(X)-\frac{l}{e} \partial_{\rho} n(X)
$$


Dans cette transformation de jauge le Lagrangien $(40)$ se récrit :

$$
\begin{aligned}
\mathscr{L} & =\frac{1}{2} \partial^{\rho} \xi(\mathrm{X}) \partial_{\rho} \xi(\mathrm{X})-\frac{1}{4} \tilde{\mathrm{F}}^{\rho \sigma}(\mathrm{X}) \tilde{\mathrm{F}}_{\rho \sigma}(\mathrm{X}) \\
& +\mathrm{m}^{2} \xi^{2}(\mathrm{X})+\left(\frac{-\mathrm{m}^{2} \mathrm{e}^{2}}{2 \lambda}\right) \tilde{\mathrm{A}}^{\rho}(\mathrm{X}) \tilde{\mathrm{A}}_{\rho}(\mathrm{X}) \\
& -\lambda \Phi_{O} \sqrt{2} \xi^{3}(\mathrm{X})-\frac{\lambda}{4} \xi^{4}(\mathrm{X}) \\
& +\mathrm{e}^{2} \tilde{\mathrm{A}}^{\rho}(\mathrm{X}) \tilde{\mathrm{A}}_{\rho}(\mathrm{X}) \xi(\mathrm{X})\left(\sqrt{2} \Phi_{0}+\frac{1}{2} \xi(\mathrm{X})\right)
\end{aligned}
$$

Lorsque $\lambda$ et e sont petits nous pouvons traiter les termes tri- et quadri- linéaires, c'est-a-dire les trois derniers termes de (42), de manière perturbative. Les quatre premiers termes de (42), qui eux sont simplement bilinéaires, constituent la partie non perturbée. De ces quatre premiers termes nous pouvons alors extraire la structure en particule de la théorie (avant perturbation par les trois derniers termes). Au champ $\xi(X)$ correspond une particule scalaire de masse $\sqrt{-2 \mathrm{~m}^{2}}$ que 1 'on appelle boson de Higgs et au champ $\tilde{A}_{\rho}(X)$ correspond une particule vectorielle de masse e $\sqrt{\frac{-m^{2}}{\lambda}}$.
Le champ $n(x)$, qui dans le paragraphe précédent correspondait au boson de Goldstone, a disparu dans le changement de jauge. En fait c'est lui qui a permis de donner une masse non nulle au champ $A_{p}(X)$. Comme précédemment, nous avons fait appara全tre la structure en particules de la théorie, mais nous avons perdu la symétrie de jauge manifeste du Lagrangien (40) par le groupe $U(1)$. Nous avons obtenu ce que nous voulions : un champ de quanta d'intéraction vectoriels massifs, à partir d'un Lagrangien invariant de jauge. Ceci a été réalisé par une brisure spontanée de la symétrie de jauge. Ce mécanisme est connu sous le nom de mécanisme de Higgs. Il est dû aussi à Englert, Brout (1964), Kibble et d'autres. Comme il a été dit plus haut le résultat important est que ce mécanisme préserve la renormalisabilité de la théorie ('t Hooft et Veltman, 1971). Ce mécanisme permet donc de formuler une théorie des intéractions faibles qui soit renormalisable et qui possède des quanta d'intéractions massifs.

5-Le modèle dit de "Weinberg-Salam".-Nous allons traiter le cas le plus simple de l'électron et de son neutrino correspondant $\nu_{e}$. Le terme d'intéraction (6) peut s'écrire:

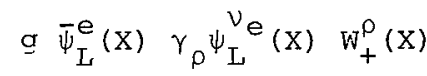

$=g \bar{\psi}_{L}(X) \gamma_{\rho}\left(\begin{array}{ll}0 & 0 \\ 1 & 0\end{array}\right) \psi_{L}(X) W_{+}^{\rho}(X)$

où nous avons pos

$$
\psi_{L}(x)=\left(\begin{array}{l}
\psi_{L}^{\nu}(x) \\
\psi_{L}^{e}(x)
\end{array}\right)
$$

I a matrice $\left(\begin{array}{ll}0 & 0 \\ 1 & 0\end{array}\right)$ peut s'écrire à I'aide des matrices de Pauli :

$$
\begin{aligned}
& \text { matrices de Pauli : } \\
& \qquad\left(\begin{array}{ll}
0 & 0 \\
1 & 0
\end{array}\right)=\sigma_{-} \\
& \sigma_{1}=\left(\begin{array}{ll}
0 & 1 \\
1 & 0
\end{array}\right) \quad \sigma_{2}=\left(\begin{array}{rr}
0 & -i \\
i & 0
\end{array}\right) \quad \sigma_{3}=\left(\begin{array}{rr}
1 & 0 \\
0 & -1
\end{array}\right) \\
& \sigma \pm=\frac{1}{2} \quad\left(\sigma_{1} \pm i \sigma_{2}\right)
\end{aligned}
$$

L'écriture (43) consiste à mettre les parties gauches de l'électron et de son neutrino dans un même doublet. Ceci suggère une invariance de jauge par le groupe SU(2). En fait on traite les intéractions faibles et électromagnétiques en même temps en considérant une invariance de jauge par le groupe $\mathrm{SU}(2) \times \mathrm{U}(1)$. Les parties droites de l'électron et de son neutrino n'apparaissent jamais dans les courants chargés faibles, on suppose donc qu'ils sont dans des singlets de $S U(2)$. En fait pour le neutrino on suppose que sa partie droite n'existe pas. Le Lagrangien libre de l'électron et de son neutrino, globalement invariant par $\mathrm{SU}(2) \times \mathrm{U}(1)$, s'écrit :

$\mathcal{L}=i \bar{\psi}_{L}(x) \gamma_{\rho} \partial^{\rho} \psi_{L}(x)+i \bar{\psi}_{R}^{e}(x) \gamma_{\rho} \partial^{\rho} \psi_{R}^{e}(x)$ ov̀ $\psi_{L}(X)$ est donné par (44).

Remarquons oue par rapport à (17) il n'y a pas de terme de masse. En effet un terme de masse de l'électron serait de la forme

$$
-m\left(\bar{\psi}_{L}^{e}(x) \psi_{R}^{e}(x)+\bar{\psi}_{R}^{e}(x) \psi_{L}^{e}(x)\right)
$$

Couplant les parties gauches et droites de l'électron, qui sont dans des représentations différentes de SU(2), il est impossible de mettre ce terme de masse sous une forme invariante par $S U(2) \times U(1)$. Maintenant rendons locale la symétrie $\mathrm{SU}(2) \times \mathrm{U}(1)$ en introduisant trois champs vectoriels $\vec{A}_{\rho}(X)$ pour $\mathrm{SU}(2)$ et un champ vectoriel $B_{\rho}(X)$ pour $U(1)$. Nous remplaçons les dérivées usuelles du Lagrangien (46) par des dérivées covariantes du type 
et nous ajoutons les termes cinétiques des champs vectoriels :

$$
\begin{aligned}
\mathcal{L} & =i \bar{\psi}_{I}(X) \gamma^{\rho}\left(\partial_{\rho}-\frac{i g}{2} \vec{A}_{\rho}(x) \vec{\sigma}_{\sigma}-i q^{\prime} B_{\rho}(x)\right) \psi_{L}(X) \\
& +i \bar{\psi}_{R}^{e}(x) \gamma^{\rho}\left(\partial_{\rho}-i g^{\prime} B_{\rho}(x)\right) \psi_{R}^{e}(x) \\
& -\frac{1}{4} \vec{F}^{\rho \sigma}(x) \vec{F}_{\rho \sigma}(x)-\frac{1}{4} B^{\rho \sigma}(x) B_{\rho \sigma}(x)(48)
\end{aligned}
$$

Examinons ce Lagrangien. Tout d'abord le terme faisant intervenir le courant charge peut s'écrire :

$$
\begin{aligned}
& \quad \frac{g}{2} \bar{\psi}_{L}(X) \gamma_{\rho}\left(A_{1}^{\rho}(X) \sigma_{1}+A_{2}^{\rho}(X) \sigma_{2}\right) \psi_{L}(X) \\
& =\frac{g}{\sqrt{2}} \bar{\psi}_{L}(X) \gamma_{\rho}\left(W_{+}^{\rho}(X) \sigma_{-}+w_{-}^{\rho}(X) \sigma_{+}\right) \psi_{L}(X) \\
& \text { où } W_{ \pm}^{\rho}(X)=\frac{1}{\sqrt{2}}\left(A_{1}^{\rho}(X) \pm i A_{2}^{\rho}(X)\right)
\end{aligned}
$$

Nous retrouvons ainsi le terme d'intéraction (43) où $g$ est remplacé par $\frac{g}{\sqrt{2}}$. La relation avec la constante de Fermi, (7), s'écrit donc dans le cas présent

$$
\sqrt{\frac{G}{2}}=8{\frac{g^{2}}{m}}_{W}^{2}
$$

Examinons maintenant les termes faisant intervenir les courants neutres. Il y a deux champs vectoriels couplés à des courants neutres : $A_{3}^{P}(X)$ et $B^{P}(X)$. Remarquons tout d'abord que si nous avions voulu traiter les intéractions faibles avec seulement une invariance de jauge de type $\mathrm{SU}(2)$, le courant neutre obtenu $n$ 'aurait fait intervenir que des parties gauches. Par conséquent cela ne peut corresponare au courant électromagnétique, qui, étant invariant par parité, fait intervenir aussi bien des parties droites que des parties gauches. Cela ne peut pas non plus corresponare au courant neutre faible opservé expérimentalement car, bien que n'étant pas invariant par parité, celuici contient aussi des parties droites. Il est donc nécessaire de considérer un groupe plus grand que $\mathrm{SU}(2)$. SU(2) $\times \mathrm{U}(1)$ est le plus petit qui convient. Il permet de traiter ensemble (et donc dans une certaine mesure d'unifier) les intéractions faibles et électromagnétiques et il prédit l'existence d'un courant neutre faible. Le champ $B^{p}(X)$ ne peut correspondre au champ électromagnétique. En effet le doublet $\left(\begin{array}{c}\psi_{L} e(x) \\ \psi_{L}^{e}(x)\end{array}\right)$ contient deux particules de charges différentes et en particulier le champ $\mathrm{B}^{\mathrm{P}}(\mathrm{X})$ est couplé au courant neutre du neutrino dont la charge électrique est nulle. En fait le champ vectoriel neutre faible $n$ 'est autre que la combinaison de $\mathrm{B}^{\rho}(X)$ et $\mathrm{A}_{3}^{\rho}(X)$ qui se couple au courant $\bar{\psi}_{I}^{\nu_{e}}(\mathrm{X}) \gamma_{\rho}{ }^{{ }^{\prime \prime}}{ }^{\nu} \mathrm{L}(\mathrm{X})$. ouant au champ électromagnétique, il correspond à la combinaison orthogonale, qui elle, n'est pas couplée au courant neutre du neutrino. Nous obtenons ainsi un mélange entre les champs $B^{\rho}(X)$ et $A_{3}^{\rho}(X)$. $L$ 'intéraction du courant neutre du neutrino s'écrit

$$
\frac{1}{2} \bar{\psi}_{L}^{\nu_{e}}(x) \gamma_{\rho} \psi_{L}^{\nu_{e}}(x)\left(g_{3}^{\rho}(x)+g^{\prime} B^{\rho}(x)\right)
$$

Le champ du boson neutre faible est donc

$$
z^{\rho}(x)=\frac{1}{\sqrt{g^{2}+g^{\prime 2}}}\left(g A_{3}^{\rho}(x)+g^{\prime} B^{\rho}(x)\right)
$$

le champ électromagnétique étant alors donné par la combinaison orthogoriale :

$$
A^{\rho}(x)=\frac{1}{\sqrt{g^{2}+g^{1^{2}}}}\left(-g^{\prime} A_{3}^{\rho}(x)+g^{\rho}(x)\right)
$$

I'angle de mélange $\theta$ apparaît en posant

$$
\operatorname{tg} \theta=\frac{g}{g}^{\prime}
$$

On peut alors faire apparaitre dans le Lagrangien (48) le terme d'intéraction électromagnétique de l'électron :

$\frac{g g^{\prime}}{\sqrt{g^{2}+g^{\prime^{2}}}}\left(\bar{\psi}_{L}^{e}(x) \gamma^{\rho} \psi_{L}^{e}(x)+\bar{\psi}_{R}^{e}(x) \gamma^{\rho} \psi_{R}^{e}(x)\right) A_{\rho}(x)$ $=\frac{g g^{\prime}}{\sqrt{g^{2}+g^{\prime 2}}} \bar{\psi}^{e}(\mathrm{X}) \gamma^{\rho} \psi^{e}(\mathrm{X}) \mathrm{A}_{\rho}(\mathrm{X})$

De la comparaison avec la formule (5) on déduit

$$
e=\frac{g g^{\prime}}{\sqrt{g^{2}+g^{\prime 2}}}=g \sin \theta
$$

les relations (50) et (56) permettent alors d'obtenir

$$
\mathfrak{m}_{W}=e\left[\frac{\sqrt{2}}{8 G}\right]^{1 / 2} \frac{1}{\sin \theta}=\frac{37,6 \mathrm{GeV}}{\sin \theta}
$$

La partie intéraction neutre faible du Lagrangien (48) s'écrit : $-\frac{g}{2 \cos \theta}\left\{\bar{\psi}_{L}^{\nu_{e}(x)} \gamma_{\rho} \psi_{L}^{\nu_{e}}(x)-\bar{\psi}_{L}^{e}(x) \gamma_{\rho} \psi_{L}^{e}(x)\right.$ $\left.+2 \sin ^{2} \theta \bar{\psi}^{e}(x) \gamma_{\rho} \psi^{e}(x)\right\} z^{\rho}(x)$

Si on écrit une intéraction courant neutrecourant neutre, par exemple pour le processus $\nu_{e} v_{e} \longrightarrow v_{e} v_{e}$ on peut dêfinir une constante $\mathrm{G}_{\mathrm{CN}}$, analogue de la constante de Fermi pour les courants neutres, :

$$
G_{\mathrm{CN}}=\frac{G}{2}\left(\frac{\mathrm{m}_{W}}{\cos \theta \mathrm{m}_{\mathrm{Z}}}\right)^{2}
$$


Le modèle de traitement des intéractions faibles et électromagnétiques qui vient d'être décrit, avec en particulier l'introduction de I'angle de mêlange $\theta$, est dû à Glashow (1961) (voir aussi Salam et Ward en 1964). Mais le Lagrangien (48) ne contient pas de termes de masses non nulles nécessaires pour les bosons $W$ et $z$ et pour l'électron. En 1967 weinberg a proposé de briser spontanément la symétrie de jauge de ce Lagrangien à l'aide du mécanisme de Higgs (voir aussi Salam en 1968). Pour cela il a introduit un doublet de champs scalaires complexes $\Phi(X)$ et a ajouté au Lagrangien (48) la quantité suivante, localement invariante par $\mathrm{SU}(2) \times \mathrm{U}(1)$, :

$$
\left[D_{\rho} \Phi(X)\right]^{+} D^{\rho} \Phi(X)-V(\Phi)
$$

où $D_{\rho}=\partial_{\rho}-i \frac{g}{2} \vec{A}_{\rho}(X) \vec{\sigma}+i \frac{g^{\prime}}{2} B_{\rho}(X)$

et $V(\Phi)=m^{2} \Phi^{\dagger}(X) \Phi(X)+\lambda\left(\Phi^{+}(X) \Phi(X)\right)^{2}$

avec $\mathrm{m}^{2}<0$. En procédant de manière analogue au paragraphe $4 \mathrm{~b}$ on fait apparaitre un terme de masse pour les champs vectoriels : $\frac{1}{3} g^{2} v^{2}\left\{2 w_{+}^{\rho}(X) w_{-} \rho(X)+\frac{1}{\cos ^{2} \theta^{2} \rho}(X) z^{\rho}(X)\right\}$

où $v=\sqrt{\frac{-m^{2}}{\lambda}}$. Ainsi :

- le boson chargé $W$ acquiert une masse

$$
m_{W}=\frac{1}{2} \mathrm{gv}
$$

- le boson neutre $\mathrm{Z}$ acquiert une masse

$$
\mathrm{m}_{\mathrm{z}}=\frac{\mathrm{gv}}{2 \cos \theta}=\frac{\mathrm{m}_{\mathrm{w}}}{\cos \theta}
$$

- quant au photon, associé au champ électromagnetique $A_{p}(x)$, il rește de masse nulle. Dans ces conditions la relation (59) devient

$$
\mathrm{G}_{\mathrm{CN}}=\frac{\mathrm{G}}{2}
$$

Remarquons de plus, que, comme dans le paragraphe $4 \mathrm{~b}$, le seuI champ scalaire réęl (boson de Higgs) qui n'a pas ëté absorbé nar les champs vectoriels, a une masse

$$
\mathrm{m}_{\varphi}=\sqrt{-2 \mathrm{~m}^{2}}=\mathrm{v} \sqrt{2 \lambda}
$$

Weinberg a aussi ajouté au Lagrangien (48) un terme de couplage de type Yukawa entre les fermions et le champ scalaire $\Phi(\mathrm{X})$ :

- $G_{e}\left\{\bar{\psi}_{\mathrm{R}}^{\mathrm{e}}(\mathrm{X}) \Phi^{\dagger}(\mathrm{X}) \psi_{\mathrm{L}}(\mathrm{X})+\bar{\psi}_{\mathrm{L}}(\mathrm{X}) \Phi(\mathrm{X}) \psi_{\mathrm{R}}^{\mathrm{e}}(\mathrm{X})\right\}$

Ce terme est localement invariant par $\mathrm{SU}(2) \times \mathrm{U}(1)$.
La procédure đu paragraphe $4 \mathrm{~b}$ apoliọuée à ce terme engendre un terme de masse pour les fermions :

$$
-\frac{G_{e} v}{\sqrt{2}} \bar{\psi}^{e}(x) \psi^{e}(x)
$$

Ainsi l'électron acquiert une masse

$$
m_{e}=\frac{G_{e}^{v}}{\sqrt{2}}
$$

tandis gue le neutrino reste de masse nulle. Tout ce qui a été fait dans ce paragraphe peut être répété de manière identique pour les couples $\left(\nu_{\mu^{-}} \mu^{-}\right)$et $\left(\nu_{\tau} \tau^{-}\right)$. De la même façon, cela peut etre fait pour les couples ( $u d$ ) (c s) et $(t b)$ en tenant compte des différents mélanges entre les guarks $a, s$ et $b$ (voir courant (4)). Le modèle qui vient d'être décrit peut alors être comparé avec l'expérience. Il y a deux types d'expériences qui permettent cette comparaison :

-1) les expériences de diffusion de neutrinos et a'antineutrinos sur la matière leptonique et hadronique.

-2) les expériences qui mesurent l'interférence entre le courant électromagnétique et le courant neutre faible. Parmi ces dernières il faut distinguer les expériences qui mesurent la violation de la parité dans les transitions atomioues et une expérience, faite au SLAC, gui mesure la violation de la parité dans la diffusion d'électrons polarisés sur une cible nucléaire.

Mis à part une certaine confusion régnant dans les difficiles experiences de physique atomique, toutes ces expériences sont d'accord pour donner la même valeur de I'angle de mélange $\theta$. La movenne des mesures actuelles est

$$
\sin ^{2} \theta=0,230 \pm 0,015
$$

De plus toutes sont d'accord pour vérifier la relation (64) ce qui semble confirmer le mécanisme de Higgs proposé par veinberó. Des relations (57) et (69) on déduit

$$
m_{-v]} \simeq 80 \mathrm{ceV}
$$

et en utilisant (63)

$$
m_{z} \simeq 90 \mathrm{GeV}
$$

Ainsi la découverte d'un boson chargé $w$ de masse approximativement $80 \mathrm{GeV}$ sera une 
confirmation de la théorie de jauge $\mathrm{SU}(2) \times \mathrm{U}(1)$. Quant à la découverte d'un boson neutre $z$ de masse approximativement $90 \mathrm{GeV}$ cela constituera une confirmation du fait que le mécanisme de brisure spontanée de la symétrie de jauge est du type de celui proposé par Weinberg. Pour être sûr qu'il s'agit d'un mécanisme de Higgs il faut découvrir l'existence d'un boson neutre de spin 0 couplé à toutes les particules massives et dont la masse est donnêe par (65). Mais $\lambda$ étant inconnu il n'est pas possible de prédire actuellement cette masse. Des estimations théoriques donnent

$$
7,3 \mathrm{GeV}<\mathrm{m}_{\varphi}<1000 \mathrm{GeV}
$$

avec une petite préférence pour $\mathrm{m}_{\varphi}$ autour de $10 \mathrm{GeV}$. La limite expérimentale actuelle est $m_{\varphi}>20 \mathrm{MeV}$. Remarquons qu'il peut $y$ exister plusieurs de ces bosons dits bosons de Higgs.

6. Conclusions.- Il existe donc un modèle, dit de "Veinberg-Salam", qui en fait constitue une vêritable théorie des intêractions faibles et qui dans une certaine mesure unifie celles-ci avec les intéractions électromagnêtiques. De plus ce mođèle est actuellement en très bon accord avec l'expérience.

Mais comme nous l'avons vu dans le paragraphe précédent, ce modèle possède deux constantes de couplages : $g$ correspondant à $\mathrm{SU}(2)$ et $\mathrm{g}^{\prime}$ correspondant à $U(1)$. Or une véritable unification demande une seule constante de couplage. Par exemple lorsque Maxwell a unifié l'électricité et le magnétisme il n'a utilisé qu'une seule constante de couplage : e. De plus ce modèle n'explique pas pourquoi la charge électrique est quantifiée; par exemple pourquoi la charge du quark d est le tiers de la charge de I'électron? Il semble que pour obtenir une veritable unification il faille traiter les intéractions êlectromagnétiques, faibles et fortes toutes les trois ensemble. Nous avons dit au paragraphe 2 que pour chacune de ses saveurs le quark possède trois couleurs différentes. Ceci est à la base d'une théorie de jauge des intéractions fortes, appelée Chromodynamique Quantique, (QCD) et basée sur une invariance locale par le grou- pe $\mathrm{SU}(3)$. Les bosons de jauge de cette intêraction sont appelés gluons. S'il n'a pas encore été possible de démontrer que cette théorie était la théorie des intéractions fortes, par contre un grand nombre de faits expérimentaux plaident en sa faveur (voir l'exposé de E de Rafael). Nous sommes en présence d'une théorie de jauge des intéractions électromagnétiques, faibles et fortes basée sur le groupe $S U(2) \times U(1) \times S U(3)$ couleur' avec trois constantes de couplage, une pour chaque sous-groupe. L'unification est réalisée en plongeant ces trois sous-groupes dans un seul groupe simple, ce qui implique une seule constante de couplage permettant de traiter les trois intéractions. C'est ce que l'on appelle la grande unification. Notons qu'une des caractéristigues de cette grande unification est de prédire une instabilité du proton, avec une durée de vie de I'ordre de $10^{31}$ années. En 1974 Georgi et Glashow ont proposé SU(5) comme groupe de grande unification; mais il $y$ en a d'autres qui sont aussi plausibles. Une autre faiblesse du modèle dit de "Weinberg-Salam" est le mécanisme de Higgs. En effet ce mécanisme fait intervenir un grand nombre de constantes de counlage arbitraires, ce qui va encore à l'encontre d'une véritable unification. De plus il apparait comme insatisfaisant à ceux qui aimeraient que les particules élementaires soient, soit des fermions de spin $1 / 2$, soit des bosons de jauge de spin 1 (en excluant la gravitation). Un mécanisme de brisure spontanée de la symétrie de jauge ne faisant pas intervenir de champ scalaire élémentaire et ayant les mêmes résultats que le mécanisme de Higgs, en particulier Ia relation (63), existe. C'est ce que l'on appelle la brisure dynamique de la symétrie de jauge. Il fait intervenir de nouveaux fermions de spin $1 / 2$ avec de nouvelles intéractions apparaissant au-dessus de $1000 \mathrm{GeV}$. Mais il n'a pas encore êté possible de construire un modèle simple avec ce type de brisure.

Pour finir un mot au sujet de l'unification avec la quatrième intéraction : la gravi- 
tation. Cela pourrait être réalisé dans ce que $I^{\prime}$ on appelle la supergravité. Il s'agit d'une théorie de jauge dans laquelle les transformations locales sont les transformations du groupe des supersymétries. Les supersymétries permettent de mettre fermions et bosons dans de même multiplets et ainsi de pouvoir transformer les uns en les autres. Si l'on considère une supersymétrie transformant un fermion en un boson et une autre supersymétrie transformant le boson en le fermion initial, le produit de ces deux supersymétries est une transformation du groupe de poincaré. On voit donc qu'en rendant locales les supersymetries on obtient une théorie qui inclue la relativité gẹnérale et donc la gravitation. Cette localisation des supersymétries introduit un doublet de quanta de jauge : le graviton de spin 2 correspondant aux transformations du groupe de Poincaré et une particule de spin $3 / 2$ appelée gravitino (il peut $\mathrm{y}$ avoir plusieurs gravitinos).

\section{References}

/1/ Abers, E.S. et Lee, B.W., Phys. Rep. C9 (1973) 1 .

/2/ Weinberg, S., Rev, Mod, Phys. 46 (1974) 255.

/3/ Iliopoulos, J., rapport jaune du CERN $\mathrm{n}^{\circ} 76-11$.

/4/ I'image de la sphère ainsi que la figure correspondante ont été empruntées à D.Z. Freedman et F. van Nieuwen-Huizen, Scientific American Vol. 238 (Février 1978) 126 . 